ACTA MYCOLOGICA

Vol. 41 (1): 99-112

2006
Dedicated to Professor Alina Skirgietto

on the occasion of her ninety fifth birthday

\title{
Co-existence and interaction between myxomycetes and other organisms in shared niches
}

\author{
IRINA O. DUDKA and KATERINA O. ROMANENKO \\ Department of Mycology, M.G. Kholodny Institute of Botany \\ National Academy of Sciences of Ukraine \\ Tereshchenkivska 2, MSP 1 Kyiv, UA 01001
}

Dudka I.O., Romanenko K.O. Co existence and interaction between myxomycetes and other organisms in shared niches. Acta Mycol. 41(1): 99 112, 2006.

The ecology of myxomycetes co existing with the Latridiidae (Coleoptera), bryophytes and ascomycetous, basidiomycetous and anamorphic fungi were studied in Crimea and at different locations on the left bank of Ukraine. Results from the left bank indicate that the Latridiidae feed on myxomycetes. Colonies of the most common 13 myxomycete species (which included Stemonitis axifera (Bull.) Macbr., S. fusca Roth, S. splendens Rost., Fuligo septica (L.) Wigg. and Mucilago crustacea Wigg.) were inhabited by 5 species of the Latridiidae. Myxomycete spores were present in guts of 19 of the 25 beetle specimens investigated. Beetles Latridius hirtus, Enicmus rugosus and $E$ fungicola seem to be obligate myxomycete feeders, while Corticarina truncatella was clearly facultative. 13 species of myxomycetes were recorded on 9 species of moss and 3 species of liverwort developing on decaying wood or bark in the Crimean Nature Reserve. Relations between myxomycetes and bryophytes on woody substrata are spatial rather than trophic, and are possibly regulated by specific microclimatic conditions inside bryophyte thallomes. 69 species of myxomycetes were found co existing with 36 species of ascomycetes, 21 species of basidiomycetes and 9 species of anamorphic fungi in the Crimean Nature Reserve. Associations formed by myxomycetes and fungi on different woody substrata are analyzed.

Key words: myxomycetes, ascomycetes, basidiomycetes, anamorphic fungi, bryophytes, Latridiidae beetles

\section{INTRODUCTION}

In terrestrial ecosystems, certain patterns can be found governing the spatial distribution of living organisms. These are connected with trophic, topic and another relations. Autotrophic plants are usually considered the foundation for the resulting consortia (ecological communities originating through co-development of heterogenous groups tightly joined by common activities), since they provide a prime food source, and a substratum on which other organisms can grow. Myxomycetes (also known as slime moulds) often form a part of those consortia, and may also make associations with various other groups of organisms (D u d ka et al. 1976). Research on 
the co-existence of slime moulds with beetles (Coleoptera), bryophytes, ascomycetous, basidiomycetous and anamorphic fungi is briefly reviewed below.

\section{BRIEF HISTORICAL REVIEW}

Myxomycetes are frequently associatiated with insects, especially beetles (Coleoptera). Stephens on et al. (1994) reviewed slime mould / beetle associations recorded since the end of the nineteenth century, and listed the beetle families most frequently forming these associations as follows: Rhizodidae, Leiodidae, Staphylinidae, Clambidae, Eucinetidae, Sphindidae, Cerylonidae, Latridiidae. The numerous and repeated records of these beetles on the same species of myxomycetes suggested an obligate connexion. Since examination of beetle gut contents usually reveals abundant myxomycete spores, it is clear the beetles feed on them. In fact, some beetle species use not only spores but also plasmodia of slime moulds as a source of nutrition (Lawrence, Newton 1980; Wheeler 1980). The term myxomycetophagy has been proposed to describe the phenomenon of beetles deriving nutrition from slime moulds (Newton 1984; Newton, Stephenson 1990).

Despite their rather diverse taxonomic range, slime moulds used by beetles as food share a number of morphological, developmental and ecological characters as follows: 1) abundant spores in large simple sporophores or large aethalia which comprise a group of sporangia covered with a common cortex and pillow-like; 2) a long period of sporophore formation during the growing season; 3) long-lasting sporophores; 4) abundance; 5) development on woody substrata; 6) spores with spiny, warty or net-like walls, 4 (5-9)-15 $\mu \mathrm{m}$ diam. (Bla ckwell 1984). Arcyria incarnata (Pers.) Pers., Stemonitis axifera (Bull.) Macbr., S. fusca Roth, S. splendens Rost., Lycogala epidendrum (L.) Fr., Stemonaria longa (Peck) Nann.-Brem., Fuligo septica (L.) Wigg., Tubifera ferruginosa (Batsch) Gmel. and others largely match those criteria. Stephens on et al. (1994) have shown that myxomycetes of the genus Stemonitis Roth are used as food by 10 beetle genera, Fuligo Hall. by 9, and Arcyria Wigg. and Tubifera Gmel. by 7 each.

The feeding preferences of some beetles for myxomycetes explains their constant association with slime moulds. There is, however, evidence that these beetles also play some part in disseminating myxomycete spores. Observation of Fuligo septica spores from Sphindidae beetle guts has shown that some spores pass through insect intestines without damage and can subsequently germinate, while transfer of spores of Physarum straminipes Lister and F. septica from the exoskeleton surface of Latridiidae beetles to agar medium has also been observed, particularly when there are large numbers of such spores (Blackwell, Laman, Gilberts on 1982).

Representatives of the family Latridiidae (Coleoptera) are small beetles, no more than $3 \mathrm{~mm}$ long, occurring under bark of fallen trunks, in decaying wood and in forest litter. Worldwide about 750 species are known, 55 in Ukraine. Typically they are associated with fungi, in particular anamorphic fungi, ascomycetes and basidiomycetes, predominantly with basidiomata of wood-destroying fungi, where they feed on the spores (Howard, Currie 1932; Newton, Stephenson 1990; Stephens on et al. 1994). Information about associations between the Latridiidae and myxomycetes has been summarized (Dudka, Trikhleb, Romanenko 2002).

The co-existence of myxomycetes and bryophytes has been studied much less than that of slide moulds and beetles. Myxomycete sporophores at the thallome 
surface of mosses and liverworts are rather widespread in nature (Stephenson, Stempen 1994; Härkönen et al. 2002; Stojanowska, Panek 2004). In most cases, however, associations of slime moulds and bryophytes arise accidentally since both components tend to occur on the same substratum - decaying and decomposing wood of deciduous and/or coniferous trees (Stephens on, Studlar 1985). It has also been surmised that myxomycetes develop more intensively and occur more frequently on fallen decaying logs overgrown with bryophytes because of their high humidity (Stojanowska, Panek 2004). Only a few myxomycetes, in particular Barbeyella minutissima Meylan, Colloderma oculatum (Lipp.) G. Lister and Lepidoderma tigrinum (Schr.) Rost. seem to be true bryophilous species. The first of these species is adapted to rich communities of liverworts, especially Novellia curvifolia and species of the genus Cephalozia which sometimes completely cover the surface of fallen decaying coniferous logs by (Schnittler, Novozhilov 1998; Schnittler, Stephenson, Novozhilov 2000; Novozhilov 2005).

In forest ecosystems many organisms are involved in decomposition of woody substrata. Apart from slime moulds, wood-inhabiting fungi are also active at various stages of succession. Some fungal species are known to develop and sporulate on the surface of myxomycete fruitbodies, using them as a feeding substratum (Roger s o n, Stephenson 1993), but that is a case of fungal parasitism on slime moulds and an example of trophic relations which are probably negative for the myxomycete. Other interrelations between myxomycetes and these fungi so far remain almost totally unstudied, although side-by-side co-existence of myxomycetes and fungi on the same woody substatum is rather commonly observed in nature. Such relations appear to be indifferent, but that is only an assumption: exact information is absent not only about the nature of those relations but about also the slime mould and fungal species compositions on which they are based. Co-existence of myxomycetes and true fungi has therefore been studied least of all (Rogerson, Stephenson 1993).

\section{OBJECTIVES}

It is clear that the characteristics of the co-existence and of interrelations between myxomycetes and other organisms is still poorly understood. The aim of the present work has therefore been to contribute to that research by identifying myxomycetes associated with Latridiidae family beetles, liverworts, mosses and wood-inhabiting anamorphic, ascomycetous and basidiomycetous fungi collected in different parts of southern Ukraine. This study has also been designed to begin analysis of relations between component members in the associations.

\section{MATERIAL AND METHODS}

Fieldwork was carried out from April to September 2000 on the left bank of Ukraine within three geobotanical regions (Donetsk, Leftbank and Starobilsk grassmeadow steppes), and in the Crimean peninsula within the Crimean Nature Reserve (Montane Crimea geobotanical region) during the growing seasons of 2000 and 2001. Left bank myxomycete collections were predominantly from forest plantations near Donetsk, and flood-plane forests around Stanychno-Luganske settlement in the valley of the Siverski Donets river (Table 1) where oak (Quercus robur L.), ash (Fraxinus 
excelsior L.) and aspen (Populus tremula L.) are dominant tree species. Beech (Fagus sylvatica L.) and Crimean pine (Pinus pallasiana D. Don) dominate other tree species in the study areas of forest communities in the Crimean Nature Reserve.

Myxomycete colonies (groups of sporophores and aethalia) were sampled in the field from fallen trunks and logs of trees in various stages of wood decay, from fallen branches of various diameters and from decaying stumps. 53 field samples of myxomycetes were collected on woody substrata the left bank study areas and 450 from the Crimean Nature Reserve. 300 samples of woody substrata from that reserve, mainly tree bark, were collected for laboratory detection of myxomycetes, using the moist chamber method (Gilbert, Martin 1933; Stephenson 1985; Nannenga-Bremekamp 1991). For myxomycete identification, the main manuals on the group were used (Nannenga-Bremek amp 1991; Novozhilov 1993; Stephenson, Stempen 1994). Slime mould nomenclature follows Ing (1999).

Of the 53 samples collected from left bank forest plantations, 25 were examined for the presence of Latridiidae in the myxomycete colonies. The biggest colonies were selected from the field samples, then divided into three more or less equal parts and placed in $90 \mathrm{~mm}$ sterile Petri dishes with filter paper at the bottom. The dark bodies of beetles emerging from the colony were easily observed against the white surface of the paper. These individuals were then identified, and their gut contents sampled. For identification of beetles the appropriate volume of Fauna Hungariae was used (Rücker 1983). Preparations of gut contents for identification of myxomycetous spores were made in Fore liquid ( $50 \mathrm{ml}$ distilled water, $20 \mathrm{~g}$ chloralhydrate, $40 \mathrm{ml}$ glycerine, $30 \mathrm{~g}$ gum-arabic).

In addition to gut content studies of Latridiidae specimens found on slime moulds collected in the field, living Latridius hirtus were kept in the laboratory on aethalia of Fuligo intermedia, to see if $L$. hirtus can complete its life cycle with only a single feed source present. Pillow-like aethalia of $F$. intermedia, $0 \cdot 5-4 \mathrm{~cm}$ long, up to 10 $\mathrm{mm}$ thick and undamaged by insects were collected in the field. These aethalia were checked for other insect inhabitants using a stereo microscope, then placed in sterile Petri dishes and stored for two weeks to ensured absence of insects. Adult Latridius hirtus individuals were then added, and the dishes were regularly examined to follow development of the successive beetle stages.

Associations between myxomycetes and bryophytes were studied in the Crimean Nature Reserve. Special attention was paid to associations where slime moulds developed sporophores, aethalia or plasmodia directly on the surface of bryophyte thallus or inside it.

Associations between myxomycetes and wood-inhabiting fungi were studied in the Crimean Nature Reserve to identify the component species and assess the influence of substratum size on species diversity.

\section{RESULTS}

\section{Interactions between myxomycetes and Latridiidae beetles on woody substrata}

Associations between myxomycetes and the Latridiidae are shown in Table 1. These associations were found to be rather widespread on the left bank of Ukraine, 
Table 1

Myxomycetes associated with Latridiidae (Coleoptera) at the left bank of Ukraine

\begin{tabular}{|c|c|c|c|}
\hline $\begin{array}{l}\text { Myxomycete } \\
\text { species, by order } \\
\text { and family }\end{array}$ & $\begin{array}{l}\text { Beetle } \\
\text { species }\end{array}$ & $\begin{array}{l}\text { Place and date of } \\
\text { association collection }\end{array}$ & Beetle gut content \\
\hline \multicolumn{4}{|c|}{ Liceales, Reticulariaceae } \\
\hline $\begin{array}{l}\text { Tubifera ferruginosa } \\
\text { (Batsch) Gmel. }\end{array}$ & $\begin{array}{l}\text { Enicmus } \\
\text { rugosus }\end{array}$ & $\begin{array}{l}\text { Lugansk oblast, s. Stan } \\
\text { ychno Luganske, 1 July } \\
2000\end{array}$ & $\begin{array}{l}\text { Spores of different myxomycete } \\
\text { species including } T \text {. ferruginosa }\end{array}$ \\
\hline \multirow[t]{3}{*}{$\begin{array}{l}\text { Reticularia } \\
\text { lycoperdon Bull. }\end{array}$} & $\begin{array}{l}\text { Enicmus } \\
\text { fungicola }\end{array}$ & Donetsk area, 6 June 2000 & Spores of $R$. lycoperdon \\
\hline & E. rugosus & Donetsk area, 26 May 2000 & Spores of $R$. lycoperdon \\
\hline & $\begin{array}{l}\text { Melanoph } \\
\text { thalma sp. }\end{array}$ & $\begin{array}{l}\text { Lugansk oblast, s. Stany } \\
\text { chno Luganske, 29 June } \\
2000\end{array}$ & $\begin{array}{l}\text { Spores of Alternaria alternata } \\
\text { (Hyphomycetes) }\end{array}$ \\
\hline \multicolumn{4}{|l|}{ Trichiales, Trichiaceae } \\
\hline $\begin{array}{l}\text { Arcyria obvelata } \\
\text { (Oeder) Onsberg }\end{array}$ & E. rugosus & $\begin{array}{l}\text { Lugansk oblast, s. Stan } \\
\text { ychno Luganske, } 30 \text { June } \\
2000\end{array}$ & Spores of $A$. obvelata \\
\hline \multicolumn{4}{|c|}{ Stemonitales, Stemonitaceae } \\
\hline $\begin{array}{l}\text { Comatricha nigra } \\
\text { (Pers.) Schroet. }\end{array}$ & E. rugosus & $\begin{array}{l}\text { Donetsk area, } 18 \text { August } \\
2000\end{array}$ & Spores of C. nigra \\
\hline $\begin{array}{l}\text { Enerthenema } \\
\text { papillatum (Pers.) } \\
\text { Rost. }\end{array}$ & E. rugosus & $\begin{array}{l}\text { Donetsk area, } 3 \text { September } \\
2000\end{array}$ & Spores of E. papillatum \\
\hline $\begin{array}{l}\text { Stemonaria longa } \\
\text { (Peck) Nann. Brem. }\end{array}$ & E. rugosus & $\begin{array}{l}\text { Donetsk oblast, Volodarski } \\
\text { district, nature reserve } \\
\text { Kamiani Mogyly, 22 July } \\
2000\end{array}$ & Spores of $S$. longa \\
\hline $\begin{array}{l}\text { Stemonitis axifera } \\
\text { (Bull.) Macbr. }\end{array}$ & E. rugosus & $\begin{array}{l}\text { Donetsk oblast, Novo } \\
\text { azovski district, Azovske } \\
\text { forestry, } 3 \text { July } 2000\end{array}$ & Spores of $S$. axifera \\
\hline \multirow[t]{2}{*}{$\begin{array}{l}\text { Stemonitis fusca } \\
\text { Roth }\end{array}$} & $\begin{array}{l}\text { Corticarina } \\
\text { truncatella }\end{array}$ & $\begin{array}{l}\text { Lugansk oblast, s. Stan } \\
\text { ychno Luganske, flood } \\
\text { plain forest, } 2 \text { July } 2000\end{array}$ & $\begin{array}{l}\text { Spores of Alternaria alternata } \\
\text { (Hyphomycetes) }\end{array}$ \\
\hline & E. rugosus & $\begin{array}{l}\text { Lugansk oblast, s. Stan } \\
\text { ychno Luganske, flood } \\
\text { plain forest, 2 July } 2000\end{array}$ & Spores of $S$. fusca \\
\hline \multirow[t]{3}{*}{$\begin{array}{l}\text { Stemonitis splendens } \\
\text { Rost. }\end{array}$} & C. & $\begin{array}{l}\text { Donetsk, } 3 \text { d city pond, } 8 \\
\text { August } 2000\end{array}$ & Spores of $S$. splendens \\
\hline & E. rugosus & $\begin{array}{l}\text { Donetsk, } 3 \mathrm{~d} \text { city pond, } 8 \\
\text { August } 2000\end{array}$ & Spores of $S$. splendens \\
\hline & $\begin{array}{l}\text { Latridius } \\
\text { hirtus }\end{array}$ & $\begin{array}{l}\text { Donetsk, } 3 \mathrm{~d} \text { city pond, } 8 \\
\text { August } 2000\end{array}$ & Spores of $S$. splendens \\
\hline $\begin{array}{l}\text { Symphytocarpus } \\
\text { amaurochaeoides } \\
\text { Nann. Brem. }\end{array}$ & E. rugosus & $\begin{array}{l}\text { Donetsk, Putylivski forest, } \\
18 \text { July } 2000\end{array}$ & Spores of S. amaurochaetoides \\
\hline
\end{tabular}


Tab. 1 cont.

\begin{tabular}{|c|c|c|c|}
\hline \multicolumn{4}{|c|}{ Physarales, Physaraceae } \\
\hline $\begin{array}{l}\text { Fuligo intermedia } \\
\text { Macbr. }\end{array}$ & E. rugosus & $\begin{array}{l}\text { Donetsk area, } 3 \text { September } \\
2000\end{array}$ & Spores of $F$. intermedia \\
\hline \multirow{3}{*}{$\begin{array}{l}\text { Fuligo septica }(\mathrm{L} .) \\
\text { Wigg. }\end{array}$} & L. hirtus & $\begin{array}{l}\text { Donetsk area, } 3 \text { September } \\
2000\end{array}$ & Spores of $F$. intermedia \\
\hline & E. rugosus & $\begin{array}{l}\text { Donetsk, Putylivski forest, } \\
18 \text { July } 2000\end{array}$ & $\begin{array}{l}\text { Spores of different myxomycete } \\
\text { species including } F \text {. septica }\end{array}$ \\
\hline & L. hirtus & $\begin{array}{l}\text { Donetsk, valley of the river } \\
\text { Bakhmutka, } 27 \text { June } 2000\end{array}$ & Spores of F. septica \\
\hline \multicolumn{4}{|c|}{ Physarales, Didymiaceae } \\
\hline \multirow[t]{2}{*}{$\begin{array}{l}\text { Mucilago crustacea } \\
\text { Wigg. }\end{array}$} & L. rugosus & Donetsk area, 10 July 2000 & $\begin{array}{l}\text { Spores of different myxomycete } \\
\text { species including } M \text {. crustacea }\end{array}$ \\
\hline & L. hirtus & $\begin{array}{l}\text { Donetsk area, } 3 \text { September } \\
2000\end{array}$ & Spores of $M$. crustacea \\
\hline
\end{tabular}

with 5 species from 4 Latridiidae genera being were found in the colonies of 13 species of myxomycetes from 10 genera.

Gut content analysis (from 5 of the Latridiidae species encountered in colonies of the 25 investigated samples of 13 myxomycete species) revealed slime moulds spores in the guts of 19 individuals (Tab. 1). In 16 of those cases, the spores found belonged to the slime mould species on which the beetle was collected. Spores of Tubifera ferruginosa, Fuligo septica and Mucilago crustacea were found in the guts of Enicmus rugosus specimens not originating from colonies of those species. Other spores distinct in morphology and size were also present in the guts of those Enicmus rugosus specimens. These may also have been myxomycete spores, but their taxonomic position was not established. No slime mould spores were found in the guts of female Melanophthalma sp. individuals found on Reticularia lycoperdon or from Corticarina truncatella individuals collected on Stemonitis fusca; instead, the guts of those beetles were filled with spores of the anamorphic fungus Alternation alternata (Fr.) Keisler.

Study of the living Latridius hirtus, kept in the laboratory on aethalia of Fuligo in termedia, resulted in the following sequence of observations: adults, laying eggs in the aethalia, larvae, moult, pupae, young adults emerging from pupae.

\section{Co-existence of myxomycetes and bryophytes on woody substrata}

Altogether 13 species of myxomycetes were recorded on 9 species of moss and 3 species of liverwort associated with woody substrata in the Crimean Nature Reserve (Table 2). Arcyria cinerea (Bull.) Pers., Echinostelium arboreum Keller et Brooks, E. minutum de Bary, Macbrideola cornea (G. Lister et Cran) Alexop., Perichaena vermicularis (Schw.) Rost., Physarum cinereum (Batsch) Pers. were most commonly encountered in association with the following bryophytes widespread in montane Crimea: Hypnum cupressiforme, Leucodon sciuroides and Porella platyphylla. Didymium 
trachysporum G. Lister on Ctenidium molluscum, Licea minima Fr. on Hypnum cupressiforme, Perichaena chrysosperma (Currey) Lister on Frullania dilata, Stemonitis fusca on Leucodon sciuroides, Symphytocarpus amaurochaetoides on Pterigynandrum filiforme, S. impexus Ing. et Nann.-Brem. on Porella platyphylla and Trichia varia (Pers.) Pers. on Anomodon viticulosus were more rare in bryophytes communities of the reserve (Tab. 2).

Augmenting information about woody substrata in Table 2, Echinostelium minutum was collected on bark of Acer stevenii Pojark., Alnus glutinosa (L.) Gaertn., Carpinus betulus L., Fagus sylvatica, Pinus kochiana Klotzsch., P. pallasiana, P. sylvestris L., Quercus petraea (Mattuschka) Liebl., Q. robur, Sorbus torminalis (L.) Crantz., Macbrideola cornea was collected on bark of Acer campestre L., Acer stevenii, Carpinus betulus, Cornus mas L., Crataegus sp., Fagus sylvatica, Fraxinus excelsior, Taxus baccata L., Tilia cordata Mill. and Perichaena vermicularis was collected on bark of Acer campestre, Alnus glutinosa, Cornus mas, Fraxinus excelsior, Pyrus communis L., Q. petraea, Taxus baccata, Tilia cordata. On the reserve Didymium trachysporum was associated with Ctenidium molluscum but there are additional records of it on fallen conifer needles, pieces of small bushes and herbivore dung. Physarum cinereum was found on fallen leaves and decaying wood, but was most frequent in bryophyte communities as listed in Table 2.

Table 2

Myxomycetes collected in the Crimean nature reserve on bryophytes

\begin{tabular}{|c|c|c|}
\hline Species of myxomycetes & Species of bryophytes & Woody substrata \\
\hline Arcyria cinerea (Bull.) Pers. & $\begin{array}{l}\text { Frullania dilatata } \\
\text { Hypnum cupressiforme } \\
\text { Porella platyphylla }\end{array}$ & absence \\
\hline Didymium trachysporum G. Lister & Ctenidium molluscum & \\
\hline Echinostelium arboreum Keller et Brooks & $\begin{array}{l}\text { Leucodon sciuroides } \\
\text { Hypnum cupressiforme }\end{array}$ & \\
\hline Echinostelium minutum de Bary & $\begin{array}{l}\text { Hypnum cupressiforme } \\
\text { Leucodon sciuroides } \\
\text { Pterigynandrum filiforme } \\
\text { Racomitrium canescens }\end{array}$ & absence \\
\hline Licea minima Fr. & Hypnum cupressiforme & decaying wood \\
\hline $\begin{array}{l}\text { Macbrideola cornea (G. Lister et Cran) } \\
\text { Alexop. }\end{array}$ & $\begin{array}{l}\text { Leucodon sciuroides } \\
\text { Porella platyphylla } \\
\text { Pterigynandrum filiforme } \\
\text { Hypnum cupressiforme } \\
\text { Metzgeria furcata }\end{array}$ & absence \\
\hline Perichaena chrysosperma (Currey) Lister & Frullania dilatata & \\
\hline Perichaena vermicularis (Schw.) Rost. & $\begin{array}{l}\text { Hypnum vaucheri } \\
\text { Porella platyphylla }\end{array}$ & absence \\
\hline Physarum cinereum (Batsch.) Pers. & $\begin{array}{l}\text { Anomodon viticulosus } \\
\text { Homolothecium sericeum } \\
\text { Hypnum cupressiforme } \\
\text { Porella platyphylla } \\
\text { Pseudoleskeella nervoza }\end{array}$ & absence \\
\hline Stemonitis fusca & Leucodon sciuroides & decaying wood \\
\hline Symphytocarpus amaurochaetoides & Pterigynandrum filiforme & decaying wood \\
\hline Symphytocarpus impexus Ing et Nann. Brem. & Porella platyphylla & \\
\hline Trichia varia (Pers.) Pers. & Anomodon viticulosus & decaying wood \\
\hline
\end{tabular}




\section{Co-existence of myxomycetes and fungi on woody substrata}

In the Crimean Nature Reserve, 69 species of myxomycetes and 66 species of fungi from the Ascomycota (36), Basidiomycota (21) and anamorphic fungi (9) were collected on decaying woody substrata of coniferous and broadleaf trees. All fungi recorded were species usually regarded as wood-inhabiting. Various complexes could be distinguished according to substratum type (Tab. 3) and range of associated species (Tab. 4).

Altogether 52 associations (Tab. 3) were encountered in the Crimean Nature Reserve between slime moulds and wood-inhabiting fungi, including 7 on large fallen trunks of trees, 34 on fallen branches of various diameters and 11 on decaying stumps. The highest numbers of myxomycete and the fungal species (from 13 to 35) occurred in associations on large fallen tree trunks. Species diversity was significantly less (from 2 to 8 ) in associations on fallen branches (from 5 to $15 \mathrm{~cm}$ in diameter) and on decaying stumps.

The following fungi were dominant (Tab. 4) in associations on small woody remnants and on large fallen decaying tree trunks. Pyrenomycetes: Ceratostomella cirrhosa (Pers.) Sacc., Lasiosphaeria hirsuta (Fr.) Ces. et de Not., Melanomma pulvis-pyrius (Pers.) Fuckel, Nectria peziza (Tode) Fr., Rosellinia conglobata (Fr. et Fuckel) Sacc.; discomycetes: Bisporella citrina (Batsch) Korf et S.E. Carp., Mollisia melaleuca (Fr.) Sacc., Orbilia coccinella (Sommerf.) Fr. These were associated with the following myxomycetes: Arcyria denudata (L.) Wettst., Ceratiomyxa fruticulosa (Mull.) Macbr., Hemitrichia clavata (Pers.) Rost., Lycogala epidendrum, Metatrichia vesparium (Batsch) Nann-Brem., Physarum nutans Pers., Stemonitis fusca, Stemonitopsis typhina (Wigg.) Nann-Brem., Trichia decipiens (Pers.) Macbr., T. scabra Rost. and T. varia (Pers.) Pers. The basidiomycete Calocera cornea (Batsch) Fr. and the anamorphic fungus Brachysporium nigrum (Link: Fr.) S. Hughes often joined those associations. Fomes fomentarius (L.: Fr.) Fr., Stereum hirsutum (Willd.) Fr., some species of the genus Xylaria Hill ex Schrank [particularly $X$. longipes Nitschke and $X$. polymorpha (Pers.) Grev.] were also found in those assocations, but only on large fallen trunks. All listed species of myxomycetes and fungi formed the main body of associations on the woody substrata in the reserve. Frequency of occurrence in associations was high throughout the period of study.

Sometimes pyrenomycetes Amphisphaeria umbrina (Fr.) de Not., Strickeria kochii Koerb., Lasiosphaeria ovina (Pers.) Ces. et de Not., discomycetes Mollisia cine-

\section{Table 3}

Distribution of associated complexes between myxomycetes and xylotrophic fungi on the different types of woody substrates

\begin{tabular}{|l|c|c|}
\hline Type of woody substrate & $\begin{array}{c}\text { Number of associated } \\
\text { complexes between } \\
\text { myxomycetes and wood } \\
\text { inhabiting fungi }\end{array}$ & $\begin{array}{c}\text { Range of species from } \\
\text { both groups in associated } \\
\text { complexes }\end{array}$ \\
\hline Large fallen tree trunks & 7 & From 13 to 35 \\
\hline Fallen branches of trees & 34 & From 2 to 6 \\
\hline Stumps & 11 & From 2 to 8 \\
\hline Total & 52 & 133 \\
\hline
\end{tabular}


Table 4

Some complexes of myxomycetes and wood inhabiting fungi on woody substrata differenti ated by size

\begin{tabular}{|c|c|}
\hline Myxomycete species & Species of wood inhabiting fungi \\
\hline \multicolumn{2}{|c|}{ Associated complexes on large fallen tree trunks } \\
\hline $\begin{array}{l}\text { Arcyria denudata } \\
\text { Fuligo septica } \\
\text { Lycogala epidendrum } \\
\text { Stemonitis fusca } \\
\text { Stemonitis flavogenita Jahn } \\
\text { Trichia scabra } \\
\text { Trichia varia }\end{array}$ & $\begin{array}{l}\text { Ceratostomella cirrhosa, C. ampullasca (Cooke) } \\
\text { Sacc., Nectria coccinea (Pers.) Fr., N. peziza, } \\
\text { Lasiospharia hirsuta, L. ovina, Melanomma } \\
\text { pulvis pyrius, Coniochaeta ligniaria (Grev.) } \\
\text { Massee, Rosellinia conglobata, Xylaria longipes, } \\
\text { Strickeria kochii, Dasyscyphus virgineus S.F. Gray, } \\
\text { Bisporella citrina, Mollisia melaleuca, Orbilia } \\
\text { coccinella, Trichophaeopsis bicuspis, Calocera } \\
\text { cornea, Fibuloporia mucida, Stereum hirsutum, } \\
\text { Fomes fomentarius, Marasmius alliaceus (Jacq.) } \\
\text { Fr., Oudemansiella sp., Armillaria mellea (Vahl.) P. } \\
\text { Kumm., Pleurothecium recurvatum, Pseudospiropes } \\
\text { subuliferus (Corda) M.B. Ellis, Acrogenospora } \\
\text { sphaerocephala (Berk. et Broome) M.B. Ellis, } \\
\text { Graphium calicioides (Berk.) Cooke et Massee } \\
\end{array}$ \\
\hline $\begin{array}{l}\text { Lycogala epidendrum } \\
\text { Metatrichia vesparium } \\
\text { Stemonitis fusca } \\
\text { Trichia varia }\end{array}$ & $\begin{array}{l}\text { Lasiosphaeria hirsuta, Nectria peziza, Melomastia } \\
\text { mastoidea (Fr.) Schroet., Melanomma pulvis } \\
\text { pyrius, Chaetosphaeria ovoidea Sacc., Mollisia } \\
\text { melaleuca, Bisporella citrina, Xylaria polymorpha, } \\
\text { Calocera cornea, Bjerkandera adusta (Willd. Ex Fr.) } \\
\text { P. Karst., Stereum hirsutum, Fomes fomentarius, } \\
\text { Pleurotus ostreatus (Jacq.) P. Kumm., Pleurothecium } \\
\text { recurvatum, Periconia cambrensis }\end{array}$ \\
\hline $\begin{array}{l}\text { Arcyria denudata } \\
\text { Comatricha laxa Rost. } \\
\text { Hemitrichia calyculata (Speg.) Farr } \\
\text { Hemitrichia clavata (Pers.) Rost. } \\
\text { Lycogala epidendrum } \\
\text { Stemonitis fusca } \\
\text { Symphytocarpus amaurochaetoides } \\
\text { Trichia scabra } \\
\text { Trichia varia }\end{array}$ & $\begin{array}{l}\text { Melanomma pulvis pyrius } \\
\text { Rosellinia conglobata } \\
\text { Lasiosphaeria hirsuta } \\
\text { Bisporella citrina } \\
\text { Trichophaeopsis bicuspis } \\
\text { Fomes fomentarius }\end{array}$ \\
\hline \multicolumn{2}{|c|}{ Associated complexes on fallen branches of trees } \\
\hline Trichia decipiens & $\begin{array}{l}\text { Ceratostomella cirrhosa, Mollisia melaleuca, Orbilia } \\
\text { coccinella, Microthelia incrustans (El. et Ev.) Corlet } \\
\text { et S. Hughes as anamorph Dendryphiopsis sp. }\end{array}$ \\
\hline Arcyria cinerea (Bull.) Pers. & $\begin{array}{l}\text { Nectria coccinea, N. peziza, Eutypa lata (Pers.) Tul. et } \\
\text { C. Tul., Orbilia coccinella, Trichophaeopsis bicuspis }\end{array}$ \\
\hline $\begin{array}{l}\text { Hemitrichia clavata } \\
\text { Trichia decipiens }\end{array}$ & $\begin{array}{l}\text { Rosellinia conglobata, Chaetosphaeria ovoidea, } \\
\text { Mollisia melaleuca, Calocera cornea }\end{array}$ \\
\hline \multicolumn{2}{|c|}{ Associated complexes on the stumps } \\
\hline $\begin{array}{l}\text { Comatricha nigra } \\
\text { Enerthenema papillatum }\end{array}$ & $\begin{array}{l}\text { Hypocrea citrina (Pers.) Fr., Mollisia lignicola Phill., } \\
\text { Stereum hirsutum }\end{array}$ \\
\hline $\begin{array}{l}\text { Ceratiomyxa fruticulosa } \\
\text { Trichia decipiens }\end{array}$ & $\begin{array}{l}\text { Lasiosphaeria hirsuta, Amphisphaeria umbrina, } \\
\text { Mollisia cinerea }\end{array}$ \\
\hline Lycogala epidendrum & $\begin{array}{l}\text { Bisporella citrina, Stereum hirsutum, Brachysporium } \\
\text { nigrum }\end{array}$ \\
\hline
\end{tabular}

rea (Batsch) P. Karst., Trichophaeopsis bicuspis (Boud.) Korf et Erb., basidiomycetes Schizopora paradoxa (Schrad.: Fr.) Donk, Phellinus ferruginosus (Schrad.: Fr.) Pat., Fibuloporia mucida (Pers.: Fr.) Niemelä, anamorphic fungi Pleurothecium recurvatum (Morgan) Höhn., Periconia cambrensis E.W. Mason et M.B. Ellis, Heteroconium chaetospira (Grove) M.B. Ellis were also present on the numerous woody remnants 
of various sizes, but these fungi were rare in comparison with the dominant species: they were often represented by a single record in the reserve.

\section{DISCUSSION}

\section{Interactions between myxomycetes and Latridiidae beetles on woody substrata}

Outside Ukraine, Reticularia lycoperdon (Liceales, Reticulariaceae), various species of the genus Stemonitis (Stemonitales, Stemonitaceae), in particular S. fusca, and more rarely Fuligo septica (Physarales, Physaraceae) are the most prevalent slime moulds in associations with the Latridiidae, while the genera Enicmus and Dienerella are the most wide-spread representatives of the family Latridiidae to be involved in associations with slime moulds (Newton, Stephenson 1990; Stephenson et al. 1994).

Within Ukraine, the same myxomycete and Latridiidae taxa were dominant in associations on the left bank. The myxomycete family Stemonitaceae (Stemonitales) was best represented, with 7 species from 5 genera, associated with 3 species of the Latridiidae, Enicmus rugosus being dominant (Tab. 1). Myxomycetes from the order Physarales were present in left bank Ukraine associations with the Latridiidae as follows (3 species from 2 genera and 2 families): Fuligio intermedia, F. septica (Physaraceae) and Mucilago crustacea (Didymiaceae). They were associated with 2 species of the Latridiidae - E. rugosus and Latridius hirtus. Both beetle species were associated with each of the 3 Physarales species. Reticularia lycoperdon (Liceales) was found from 3 locations in association with Enicmus fungicola, E. rugosus and Melanophthalma sp. (distinguenda-group - an exact species identification was not possible from the females collected). Interestingly, $R$. lycoperdon has been widely reported as the basic myxomycete component in associations with the Latridiidae in many parts of the world.

Among the myxomycetes connected with Latridiidae beetles in our left bank sampling sites, large aethalia $(0 \cdot 5-20 \mathrm{~cm}$ long and $0 \cdot 1-3 \mathrm{~cm}$ thick) are typical for Reticularia lycoperdon, Fuligo intermedia, F. septica and Mucilago crustacea. Sporophores closely grouped in large and dense colonies are observed in species of the genus Stemonitis (especially S. fusca and S. splendens). Sporophores of Symphytocarpus amaurochaetoides tend to coalesce resulting in the formation of complex fruiting structures. The sporophores and aethalia of these slime moulds therefore provide sufficient spores to attract feeding by $L a$ tridiidae beetles. Together with Stemonaria longa, Comatricha nigra and some other species, they all have a long period of sporophore formation and the sporophores so formed then tend to be long-lasting. Furthermore, all of these slime mould species develop on woody substrata and have spores from 4-15 $\mu \mathrm{m}$ diam. with ornamented walls. They therefore have morphological, developmental and ecological characters which fit the criteria proposed by Blackwell (1984) for slime moulds associated with beetles.

The presence of myxomycete spores in Latridiidae gut contents shows that these beetles eat not only true fungi but also myxomycetes, while our observations of $\mathrm{La}$ tridius hirtus kept alive in the laboratory in defined conditions showed that at least one species is able to complete its life cycle, apparently normally, with only aethalia of one myxomycete species, $F$. intermedia, as food. 
There are very few reports from other parts of the world of myxomycete feeding by Latridiidae beetles. A few individuals of an unknown species of Enicmus were collected on colonies of Reticularia lycoperdon from four locations in Himachal Pradesh (India): the gut contents of two were analysed and spores of $R$. lycoperdon found in both. A few individuals of a previously undescribed species of Dienerella were discovered on colonies of Stemonitis fusca from two locations in Himachal Pradesh: the guts content of an individual from each location were examined, and in both cases the guts were filled with spores of $S$. fusca (N ew t on, S t e p h e n s on 1990). Our results on for Latridiidae beetles and myxomycetes in Ukraine are entirely compatible with those observations. Our work therefore confirms that, in addition to the Cerylonidae, Leiodidae, Scaphidiinae (Staphylinidae), Sphindidae and some other beetle families, the Latridiidae also feed on myxomycetes.

There is some question about whether the Latridiidae are obligate or facultative feeders on myxomycetes. Newton and Stephens on (1990) considered their new species of Dienerella from India to be a facultative feeder. Results from the left bank of Ukraine suggest that Latridius hirtus, Enicmus rugosus and E. fungicola may be obligate feeders: L. hirtus, in particular, has been shown to be capable of completing its life cycle with only myxomycetes as a food supply. Corticarina truncatella, however, is more likely to be facultative: records on myxomycetes are rather rare, and it was usually found in forest litter, and on hay and other plant remains; furthermore gut content analysis shows this species can feed on fungal spores as well as those of slime moulds.

Use of myxomycetes by Latridiidae beetles extends beyond feeding: they may lay their eggs in sporophores, aethalia and plasmodia; large downy colonies of myxomycetes may provide good cover for larvae and adults. The absence of myxomycete spores in guts of Melanophthalma sp. distinguenda-group beetles from colonies of Reticularia lycoperdon suggests that the association between these beetles and myxomycetes is based on something other than a food chain - perhaps a spatial relationship - although that needs further study.

\section{Co-existence of myxomycetes with bryophytes on woody substrata}

Decaying fallen trunks, logs, large branches, decomposing stumps are good substrata for simultaneous development of a diverse biota, including slime moulds, liverworts and mosses. Myxomycetes often occur on wood overgrown with bryophytes (Stephenson, Studlar 1985; Stephenson, Stempen 1994; Schnittler, Novozhilov 1998; Schnittler, Stephenson, Novozhilov 2000; Härkönen et al. 2002). Woody substrata covered with bryophytes provide favourable conditions for development of slime moulds, particularly on account of their high humidity (Stojanowska, Panek 2004; Novozhilov 2005).

All slime mould species found in the reserve associated with mosses and liverworts are usually considered lignicolous or corticolous: Licea minima, Stemonitis fusca, Symphytocarpus amaurochaetoides and Trichia varia, usually collected from decaying wood (Ing 1999), were also found on that substratum in the reserve. Arcyria cinerea, Echinostelium arboreum, E. minutum, Macbrideola cornea, Perichaena vermicularis and Physarum cinereum are known as typical corticolous species (Mitchell 1980). In our study area most of these corticolous species also were more often noted on 
bark of various trees (Tab. 2). Echinostelium arboreum was the exception. In the reserve, every collection was made on moss, predominantly Leucodon sciuroides but once Hypnum cupressiforme.

Didymium trachysporum and Physarum cinereum are well documented as species developing on plant remains in the litter (Ing 1999), but there is also a report of their occurrence in association with bryophytes (Stephenson, Studlar 1985). Our results from Crimea match this pattern exactly. Some slime mould species ( $A r$ cyria cinerea, Echinostelium arboreum, E. minutum, Macbrideola cornea, Perichaena vermicularis and Physarum cinereum) developed on more than one bryophyte species. This may be evidence that some associations between myxomycetes and bryophytes are purely accidental, arising because slime moulds and bryophytes are both well-adapted to develop on the same substrata (bark or wood). The associations may, however, alternatively arise because bryophytes provide ideal protection and other ecological conditions in which slime moulds can complete their full life cycle from plasmodia to sporophores.

The relation between myxomycetes and bryophytes can be regarded as one of shared space. The bryophytes ensure temperature and humidity conditions suitable for slime mould development. Fluctuations of temperature and humidity are less inside the bryophyte thallomes than outside, so that the bryophytes function like a moist chamber for the slime moulds, providing favourable conditions for their development. This function benefits not only the myxomycetes but also the bacteria, algae and protozoa on which they feed (Stephenson, Stempen 1994). The myxomycetes produce sporophores and aethalia from plasmodia in the drier areas on the bryophyte open surfaces. Bryophytes associated with myxomycetes thus seem neither to benefit nor be damaged by them (Stephen son, Studlar 1985): bryophytes associated with slime moulds continue normal growth and reproduction, while the myxomycetes have a favourable biotope in the bryophytes communities. Interrelations between the bryophytes and the slime moulds are thus generally positive and may be classified as commensal: unilateral use of one species by another without damage.

\section{Co-existence of myxomycetes and fungi on woody substrata}

Large fallen tree trunks might be thought to provide the richest associations of slime moulds and wood-inhabiting fungi, but in fact most associations (34) were recorded on fallen branches (Tab. 3). Direct contact of fallen branches with soil allows water to accumulate in the wood. Shade and grass cover makes loss of humidity from fallen branches and temperature fluctuations in the lower layer of forest vegetation less than from large fallen trunks and stumps. The hydrothermal regime of the fallen branches and their location positively influenced formation of a significant number of associations between myxomycetes and wood-inhabiting fungi. Such associations on woody substrata of various sizes and volumes are not directly connected and can exist independently. The biotic relations within those associations can therefore be characterized as neutral.

Species diversity in myxomycete / fungal associations on woody substrata can be compared according to size and volume of the substrata sampled (Tab. 4). One possible explanation for the correlation between species diversity and size (diameter) 
of the woody substratum may be that larger woody remnants conserve water longer thus providing both myxomycetes and wood inhabiting fungi with better conditions for development. Substratum size may also determine the amount and availability of nutrients necessary for growth and formation of fruitbodies.

\section{CONCLUSIONS}

Co-existence and interaction between slime moulds, beetles, bryophytes and fungi have specific features for every pair of interacting organisms: at one end, the Latridiidae may be either obligate or facultative feeders on slime moulds; at the other, relations between slime moulds and wood-inhabiting fungi are neutral. Further studies are necessary to clarify the mechanisms regulating these biotic relations.

Acknowledgements: We would like to thank Dr Tetiana Trikhleb from the National University in Donetsk (Ukraine) for identification of Latridiidae, and Dr Svitlana Nyporko from the Department of Lichenology and Bryology, M.G. Kholodny Institute of Botany NASU in Kyiv (Ukraine) who helped with identifica tion of bryophytes. Our special thanks to David Minter (Egham) for his critical reading the paper and checking English.

\section{REFERENCES}

Bl a ckwell M. 1984. Myxomycetes and their artropod associates. Fungus Insect Relationship: Perspec tives in Ecology and Evolution. N.Y. Columbia Univ. Press: 6790.

Blackwell M., La man T.G., Gilberts on R.L. 1982. Spore dispersal of Fuligo septica (Myxo mycetes) by latridiid beetles. Mycotaxon 14:58 60 .

Dudka I.O., Smitska M.F., Smyk L.V., Merezhko T.O. 1976. Some theoretical prob lems of mycocoenology. II. Consotria and the role of fungi in consortive relations. Ukr. Bot. J. 33 (2): 113124 (in Ukrainian).

Dudka I.O., Trikhleb T.A., Romanenko K.O. 2002. Associations of myxomycetes with the beetles (Coleoptera, Latridiidae). Ecol. and Noosphaerol. 12 (3 4): 5464 (in Ukrainian).

Gilbert H., Martin G. 1933. Myxomycetes found on bark of living trees. Univ. Iowa Stud. Nat. Hist. 15: 38 .

Härkönen M., Rikkinen J., Ukkola T., Enroth J., Virtanen V.etal. 2002. The com munities of bryophytes, lichens and myxomycetes on bark of living trees in Hunan, Southern China. 4 th Intern. Congress on Systematics and Ecology of Myxomycetes (August 4 9, 2002). Abstract. Scripta Bot. Belg. 22: 3637.

Howard E.L., Currie M.E. 1932. Parasitism of myxomycete plasmodia on the sporophores of hymenomycetes. J. Arnold Arboretum 13: 270281.

Ing B. 1999. The Myxomycetes of Britain and Ireland. An Identification Handbook. The Richmond Publishing Co. Ltd. 374 pp.

Lawrence J.F., Newt on A.F. 1980. Coleoptera associated with the fruiting bodies of slime molds (Myxomycetes). Coleopterests Bull. 14: 129143.

Mitchell D.W. 1980. A Key to the Corticolous Myxomycetes. Cambridge, England: The Brit. Mycol. Soc. $63 \mathrm{pp}$.

Nannenga Bremekamp N.E. 1991. A Guide to Temperate Myxomycetes. Bristol, England: Bio press Ltd. 409 pp.

New t on A.F. 1984. Mycophagy in Staphylinoidea (Coleoptera). Fungus Insect Relationship: Perspec tives in Ecology and Evolution. N.Y. Columbia Univ. Press: 302353.

Newton A.F., Stephenson S.L. 1990. A beetle/slime mold assamblage from Northern India (Coleoptera; Myxomycetes). Oriental Insects 24: 197218.

Novozhilov Yu.K. 1993. An Identification Handbook of Russia. Division Slime Moulds. I. Class Myxomycetes. SPb: Science. 288 pp. (in Russian).

Novozhilov Yu.K. 2005. Myxomycetes (class Myxomycetes) of Russia: taxonomic composition, ecology and geography. Autorev. of Dr of biol. thesis. 48 pp. (in Russian). 
Rogerson C.T., Stephenson S.L. 1993. Myxomyceticolous fungi. Mycologia 85 (3): 456469.

R ü cke r W.H. 1983. Bunkóscsápi Bogarak VII, Clavicornia VII: Merophysiidae, Latridiidae, Dasyce ridae. Magyarország Állatvilága (Fauna Hungariae) 158. Budapest. 68 pp.

Schnittler M., Novozhilov Yu.K. 1998. Late autumn myxomycetes of the northern Ammer gauer Alps. Nova Hedwigia. 66 (1 2): 205222.

Schnittler M., Stephenson S.L., Novozhilov Yu.K. 2000. Ecology and world distribu tion of Barbeyella minutissima (Myxomycetes). Mycol. Res. 104: 15181523.

St e p h e n s on S.L. 1985. Slime molds in the laboratory II. Moist chamber cultures. Amer. Biol. Teach er 47: 487489.

Stephens on S.L., Stempen H. 1994. Myxomycetes: A Handbook for Slime Molds. Portland, Oregon: Timber Press. 183 pp.

Stephenson S.L., Studlar S.M. 1985. Myxomycetes fruiting upon bryophytes: coincidence or preference? J. Bryol. 13: 537548.

Stephenson S.L., Wheeler Q.D., McHugh J.V., Frassinet P.R. 1994. New North American associations of Coleoptera with Myxomycetes. J. Nat. Hist. 28: 921936.

Stojanowska W., Panek E. 2004. Myxomycetes of the nature reserve near Wałbrzych (SW Po land) Part II. Dependence on the substrate and seasonality. Acta Mycol. 39 (2): 147159.

Whe ele r Q.D. 1980. Studies on Neotropical slime mold/beetle relationships. Part I. Natural history and description of a new species of Anisotoma from Panama (Coleoptera: Leiodidae). Proc. Ento molog. Soc. Washington 82: 493498.

\section{Wspólistnienie i interakcje pomiędzy śluzowcami a innymi organizmami}

\section{Streszczenie}

W artykule podane są wyniki badań mikoekologicznych na terenie Krymu i Ukrainie Le wobrzeżnej. Określono status taksonomiczny śluzowców, które współistnieją z owadami z ro dziny Latridiidae (Coleoptera), mchami, grzybami: Ascomycota, Basidiomycota i grzybami anamorficznymi. Badania stosunków śluzowców i owadów z rodziny Latridiidae w stepowych regionach Ukrainy Lewobrzeżnej ukazały istnienie troficznego związku owadów i śluzowców. W koloniach 13 gatunków śluzowców, wśród których przeważały: Stemonitis axifera, S. fusca, S. splendens, Fuligo septica i Mucilago crustacea, występowało 5 gatunków owadów z rodziny Latridiidae. Analiza treści żołądkowej ukazała istnienie zarodników śluzowców w przewodach pokarmowych 19 z 25 przebadanych osobników. Stwierdzono, że owady Latridium hirtus, En cimus rugosus, E. fungicola są myxomycetofagami obligatoryjnymi, a Corticarina truncatella może być uważany za myxomycetofaga fakultatywnego. 12 gatunków śluzowców podanych było z powierzchni 12 gatunków mchów, które rozwjiały się na butwiejącym drewnie lub na korze w Krymskim Rezerwacie Przyrody. Stosunki śluzowców i mchów, wspólnie rosnących w Rezerwacie, na jednym drzewnym substracie są scharakteryzowane jako topiczne. Są one regulowane przez specyficzne mikroklimatyczne warunki, które tworzą się w środku mchów z wysoką wylgotnością. Stwierdzono wspólne występowanie 69 gatunków śluzowców z 36 ga tunkami workowców, 21 gatunkami podstawczaków, 9 gatunkami anamorficznych grzybów. Analizowany skład asocjacji, które tworzą śluzowce z grzybami zależy od rozmiarów i objęto sci substratów drzewnych. 